\title{
Drive optimisation of a pulsatile Total Artificial Heart"
}

\author{
André Pohlmann $^{1}$, Marc Lessmann ${ }^{1}$, Thomas Finocchiaro ${ }^{2}$, Andreas Fritschi $^{2}$, \\ ULRICH STEINSEIFER ${ }^{2}$, THOMAS SCHMITZ-RODE ${ }^{2}$, KAY HAMEYER ${ }^{1}$ \\ ${ }^{1}$ RWTH Aachen University, Institute of Electrical Machines \\ Schinkelstraße 4, D-52056 Aachen, Germany \\ e-mail: Andre.Pohlmann@iem.rwth-aachen.de \\ ${ }^{2}$ RWTH Aachen University, Chair of Applied Medical Engineering \\ Pauwelsstraß 20, D-52074 Aachen, Germany \\ e-mail:finocchiaro@hia.rwth-aachen.de
}

(Received: 02.07.2010, revised: 08.12.2010)

\begin{abstract}
Total Artificial Hearts (TAHs) are required for the therapy of terminal heart diseases as heart transplants are only a limited option due to the available number of donor hearts. For implantation TAHs have to meet constraints regarding its dimensions, weight, perfusions and electrical losses. An innovative linear driven TAH is presented, which meets all constraints except weight. Therefore the geometry of the linear drive is optimised to reduce its weights while simultaneously limiting the electrical losses as much as possible. In order to calculate the losses, this paper introduced a combined calculation chain consisting of FEM simulations and analytical equations. Based on this chain the linear drive is optmised by the method of parameter variations. The results yield a hierachic order of parameters which are most suitable for the weight reduction of the drive for low losses. By this the weight of the linear drive is reduced by $25 \%$. As the allowable loss limit is not exceeded yet, room for further weight reduction achieved by an optimisation of the axial geomtry parameters is given.
\end{abstract}

Key words: Total Artificial Heart, TAH, linear drive, optimisation, losses

\section{Introduction}

For the therapy of terminal heart diseases, heart transplants are only a limited option due to the available number of donor hearts. This is why Total Artificial Hearts (TAHs) are required for destination therapy, which can sustain the patient's life. Nowadays, there is only one TAH system with clinical approvement available [1], which has the disadvantage of extra corporal connections through the human abdominal wall, increasing the risk of infections. Furthermore, the patient's quality of life is restricted to a minimum due to the high amount of extra corporal periphery. In order to reduce this risk of infections and to maintain the patient's quality of life,

* This is extended version of a paper which was presented at the 21st Symposium on Electromagnetic Phenomena in Nonlinear Circuits, Essen-Dortmund, 29.06-02.07, 2010. 
a totally implantable TAH, supplied via a transcutaneous power transfer system, is desirable. A total implantable TAH system has rigorous restrictions in outer dimensions and weight due to the limited space in the human thorax. Additionally, blood damage caused by overheating and high shear stress must be prevented, limiting the thermal losses of the drive and setting high demands to the blood pump design. On the other side the TAH has to pump up to 6 litre of blood per minute against a medium aortic pressure of $100 \mathrm{mmHg}$. The following list of constraints for TAH implantation, based on previous investigations and literature researches, has to be achieved:

a) due to the limited space in the human thorax, the dimensions of the TAH should not exceed $85 \mathrm{~mm}$ in diameter and $95 \mathrm{~mm}$ in length;

b) the total weight of the TAH should be less than $800 \mathrm{~g}$, compared to the $400 \mathrm{~g}$ of a natural heart;

c) the average pumping capacity should amount to $61 /$ min against a medium aortic pressure of $100 \mathrm{mmHg}$ and provide an additional overload capacity;

d) the electrical losses occurring in the drive have to be less than $20 \mathrm{~W}$ to avoid blood damage due to the heat tolerance of the human body;

e) A linear drive has to provide a maximum force of $70 \mathrm{~N}$;

f) A long durability ( $>5$ years) of the TAH by a wear free and/or redundant system is desirable.

In [2] the development of a TAH (Fig. 1) and its proof of concept are presented. This TAH concept is driven by a linear motor, which is located in the middle of the blood pump.

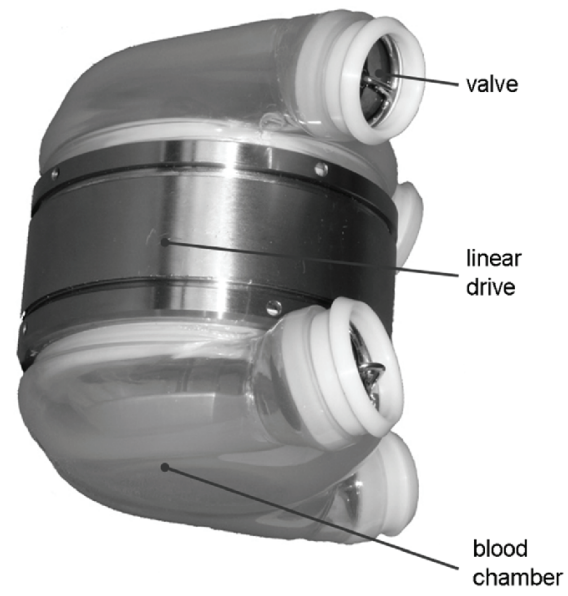

Fig. 1. Total Artificial Heart

On each side of the motor a blood chamber with a capacity of $60 \mathrm{ml}$ is attached. These chambers are compressed alternating by two pusher plates, which are directly connected to the linear drive. This arrangement yields a pulsatile blood flow, which flow direction is determined by the valves at the inlet and outlet of the blood chambers. Thus, 100 beats per minute (bpm) theoretically provide the required perfusion of $61 / \mathrm{min}$. However, measurements and in vivo tests revealed that this TAH provides only a flow rate of $61 /$ min against a medium aortic 
pressure of $100 \mathrm{mmHg}$ at a pumping frequency of $150 \mathrm{bpm}$. Possible reasons are flow losses in the valves, the insufficient stiffness of the blood chamber housing, as well as the not completely evacuated blood chamber during one beat cycle. In [3] it is assumed that an overheating of the blood can be prevented by limiting the electric losses in the drive to $20 \mathrm{~W}$. In order to validate this assumption, thermal measurements have been performed on the TAH prototype. While pumping against an medium aortic pressure of $100 \mathrm{mmHg}$ with a frequency of $150 \mathrm{bpm}$, the surface temperature in the region of the diaphragm, which is one of the thermal hotspots, rises only about $1{ }^{\circ} \mathrm{C}$. During testing in this nominal operation point the average electric losses have been $11 \mathrm{~W}$ for the drive and the converter, indicating that blood damage caused by overheating is unlikely and even $20 \mathrm{~W}$ of thermal losses can be dissipated to the blood and the surrounding tissue. The dimensions of the TAH are within the predefined limits (a), but its weight of $1139 \mathrm{~g}$ exceeds the given limit of $800 \mathrm{~g}$ (b). With more than $600 \mathrm{~g}$ the drive is the heaviest component of the TAH. For this reason this paper focuses on the weight reduction of the TAH by applying an optimisation chain.

\section{Drive optimisation}

The assembly of the TAH drive is shown in Figure 2.
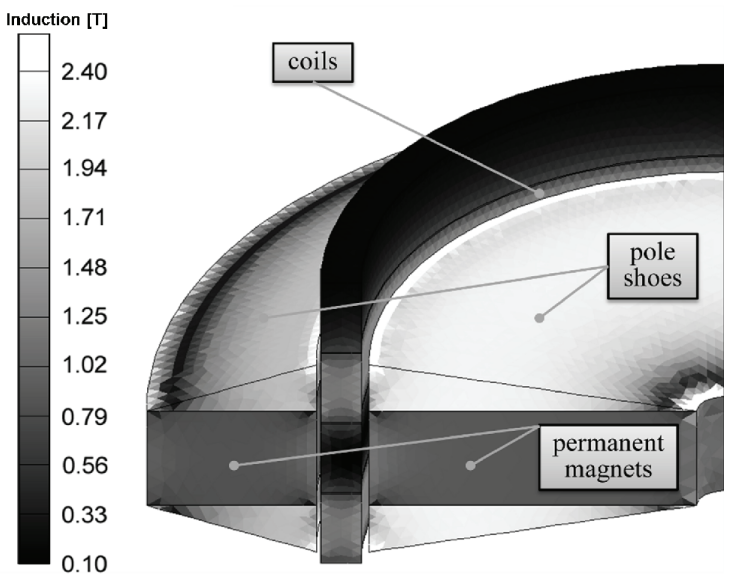

Fig. 2. Flux density distribution in $\mathrm{TAH}$

It is excited by an inner and an outer permanent magnet ring, made of Neodymium Iron Bor $(\mathrm{NdFeB})$. Due to the dimensions of the outer permanent magnetic ring $\left(d_{\text {out }}=85 \mathrm{~mm}\right)$, the maximum achievable remanent flux density is only $1.35 \mathrm{~T}$, while the remanent flux density of the inner magnet amounts to $1.4 \mathrm{~T}$. Above and beyond the magnets soft magnetic pole shoes are fastened, made of an iron vanadium cobalt alloy with the highest achievable saturation induction of $2.4 \mathrm{~T} \mathrm{[4],} \mathrm{concentrating} \mathrm{the} \mathrm{flux} \mathrm{density} \mathrm{in} \mathrm{the} \mathrm{air} \mathrm{gap.} \mathrm{The} \mathrm{coils,} \mathrm{arranged} \mathrm{in} \mathrm{the}$ air gap between the permanent magnet rings and the pole shoes, are hand wounded with a rectangular copper wire. Thus, a copper fill factor of about $75 \%$ is achieved. When supplying the coils with a dc current, they are moving according to the Lorentz force equation. 
The objective of the drive optimisation is to decrease the weight of the drive while not exceeding the electric loss limit (d). Due to the use of magnetic and electric high end materials, the electric losses are at its minimum at the starting point of the drive optimisation. As the weight reduction can only be achieved by variations in the geometry of the drive, yielding a reduced permanent magnet volume or reduced active wire length of the coils, the losses will rise during the optimisation process.

\subsection{Measurement of the force vs. displacement curve}

In order to determine the required forces for compressing the blood chambers during one beat cycle, the measurement of the force vs. displacement curve at nominal operation has to be performed for the optimisation. Therefore the left blood chamber, supplying the arterial circuit, is connected to a circulatory mock loop, simulating the pressures in the arterial human blood circuit. Due to the higher pressures in the arterial circuit compared to the pulmonary circuit, the highest required forces are observed for sufficient blood perfusion here. For this measurement the linear motor is replaced by a modified milling machine, driving the pusher plate, which evacuates the blood chamber, of the arterial circuit. During the sinusoidal movement of the pusher plate with an amplitude of $9.25 \mathrm{~mm}$ at frequencies between $80 \mathrm{bpm}$ and $160 \mathrm{bpm}$, the required forces for driving the plate are recorded. Additionally, systolic and diastolic pressures as well as the flow rates at the in- and outlet valves are measured. For the left heart chamber the minimum required blood flow of $61 /$ min against a medium aortic pressure of $100 \mathrm{mmHg}$ is already achieved at a frequency of $120 \mathrm{bpm}$ (Fig. 3), due to improvements of the stiffness of the blood chamber housing when compared to the blood chamber used for the previous prototype.

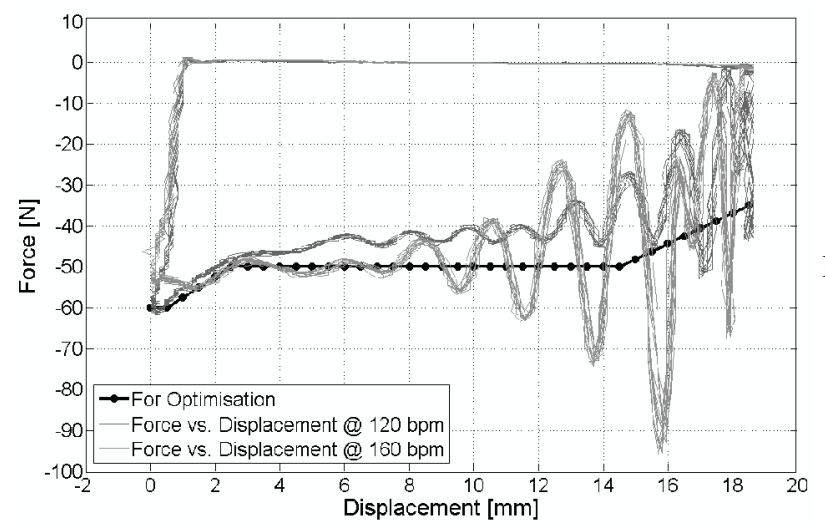

Fig. 3. Force vs. displacement

At this frequency the required force at the displacement of $-18.5 \mathrm{~mm}$ reaches the maximum force of $60 \mathrm{~N}$ for all measurements. When comparing this force with the constraints list, presented in the introduction, the requirements (e) have changed. On the other side the amplitude of the force fluctuations at the beginning reaches its maximum at a frequency of 160 bpm. These fluctuations are caused by oscillations of the flexible diaphragm and the fluid between the valves and the pusher plates, induced by the hard position control of the testing 
machine. In combination with the linear drive, these oscillations will be damped due to the limitation of achievable forces of the drive. Hence, the flattened curve represents the average forces required at any position to achieve the blood perfusion for nominal operatioin.

\subsection{Calculation of the weight and the electric losses}

For the optimisation the weight and the resulting losses have to be computed. The total weight of the drive can be calculated by multiplying the volumes, extracted from CAD models, with their material densities and accumulating the resulting weights. As the electric losses are dependent on the dc current supply of the coils, which itself depend on the required forces and the flux density distribution in the drive, a computation chain is needed for their determination.

Because of the air gap width of about $4 \mathrm{~mm}$ between the inner and outer permanent magnet ring, the counter effect of the field, excited by the coils, can be neglected. Additionally, iron losses in the pole shoes and permanent magnets are negligible at the nominal operation frequency of $2 \mathrm{~Hz}$. Hence, the ohmic losses for each coil are dominating and computed analytically by the product of the resistance, also extracted from CAD models, and the coil currents, which are calculated as follows.

As the magnetic leakage flux is significant and the iron vanadium cobalt alloy is magnetically saturated, Figure 2, non linear Finite Element (FE) simulations, performed by a $3 \mathrm{~d}$ static problem-solver from the iMOOSE package [5], which contains FE solvers developed at the Institute of Electrical Machines of the RWTH Aachen University, are required for the computation of the flux density distribution in the air gap. To reduce the computation time especially for further parameter variation, a combination of analytical and numerical approaches is applied to this optimisation. Therefore, the current distribution of the coil supplies and the resulting actuator forces are computed analytically, based on the FE field computations of the average radial flux density distribution $\bar{B}_{r, n}(x)$ in the air gap, penetrating each coil $n$ at their axial displacement $x$. Due to the negligible counter effects of the coils' field, force computations are performed analytically by means of Lorentz force equation [3]:

$$
F_{n}(x)=I_{n}(x) \cdot l \cdot \bar{B}_{r, n}(x) .
$$

In the linear drive the wire length 1 is identical for all four coils. If all coils are supplied with the same dc current $I_{n}(x)$, the generated force of each coil $F_{n}(x)$ is proportional to the average radial amount of flux density $\bar{B}_{r, n}(x)$, penetrating each coil at a given axial displacement $x$. Therefore, the ratio of the average flux density $\bar{B}_{r, n}(x)$ penetrating one coil to the average flux density $\bar{B}_{r}(x)$ penetrating all coils can be determined with the current factors $k_{I}(x)$ for each displacement.

$$
k_{I, n}(x)=\frac{\bar{B}_{r, n}(x)}{\sum_{n=1}^{4}\left|\bar{B}_{r, n}(x)\right|} .
$$

As the allowable ohmic losses are limited, the coil currents are limited as well. In order to achieve a maximized forces generation of all coils under the constraint that the sum of their 
current supplies $I_{\text {sum }}(x)$ is limited, all coils should be supplied according to their current factor $k_{I, n}$.

$$
I_{n}(x)=k_{I, n}(x) I_{\text {sum }}(x) .
$$

The required force $F_{\text {required }}(x)$, which is obtained from the predefined force displacement curve, Figure 3, has to be provided by all coils.

$$
F_{\text {required }}(x)=\sum_{n=1}^{4} I_{n}(x) \cdot l \cdot \bar{B}_{r, n}(x)
$$

When inserting equation 3 in equation 4 and transforming the result to

$$
I_{\text {sum }}(x)=\frac{F_{\text {required }}(x)}{\sum_{n=1}^{4} \bar{B}_{r, n}(x) \cdot k_{I, n}(x) \cdot l},
$$

the required coil supply to generate the required forces can be determined. Finally, the resulting position dependent ohmic losses $P_{\text {sum }}(x)$ are determined by accumulating the losses of each coil, which are calculated by multiplying the square of the coil's current $I_{n}(x)$ with its resistance $R$. To compute the average losses during one beat cycle, the maximum axial displacement of the pusher plates of $18.5 \mathrm{~mm}$ is devided into segments with a length of $0.5 \mathrm{~mm}$ :

$$
x_{m}=m \cdot 0.5 \mathrm{~mm} \quad m=0, \ldots 37 .
$$

For each of these $m$ segments the delay time $t_{m}$ of the pusher plates within each segment is calculated, based on the assumption of a sinusoidal movement of the pusher plates:

$$
t_{m}=\frac{\arcsin \left(\frac{x_{m+1}-9.25 \mathrm{~mm}}{9.25 \mathrm{~mm}}\right)-\arcsin \left(\frac{x_{m}-9.25 \mathrm{~mm}}{9.25 \mathrm{~mm}}\right)}{2 \cdot \pi \cdot f} .
$$

Finally, the position dependent losses are weighted with the time factor tm for the calculation of the average losses during one beat cycle: As the delay time tm differs for each displacement due to the sinusoidal movement, the position dependent losses are weighted with $t_{m}$. By multiplying the weighted losses with the pumping frequency $f$ the average losses $P_{\text {sum }}(x)$ are determined

$$
P_{\mathrm{sum}}(x)=f \cdot \sum_{m=0}^{36} P_{\mathrm{sum}, f w}\left(x_{m}\right) \cdot t_{m}+P_{\mathrm{sum}, b w}\left(x_{m}\right) \cdot t_{37-m}
$$

The medium pressure of the polmunary blood circuit $(27 \mathrm{mmHg})$ is lower than in the arterial blood circuit $(100 \mathrm{mmHg})$. Therefore the required forces and the resulting losses are lower in this case as well. For this reason the average electric losses $P_{\text {sum }}(x)$ are the sum of the resulting losses when evacuating the arterial blood chamber $\left(P_{\text {sum; } f_{w}}\right)$ and the pulmonary blood chamber $\left(P_{\text {sum; } b w}\right)$. Based on this tool chain, the drive is optimised by a combination of analytical and numerical computations to achieve accurate results in a minimum of computation time. 


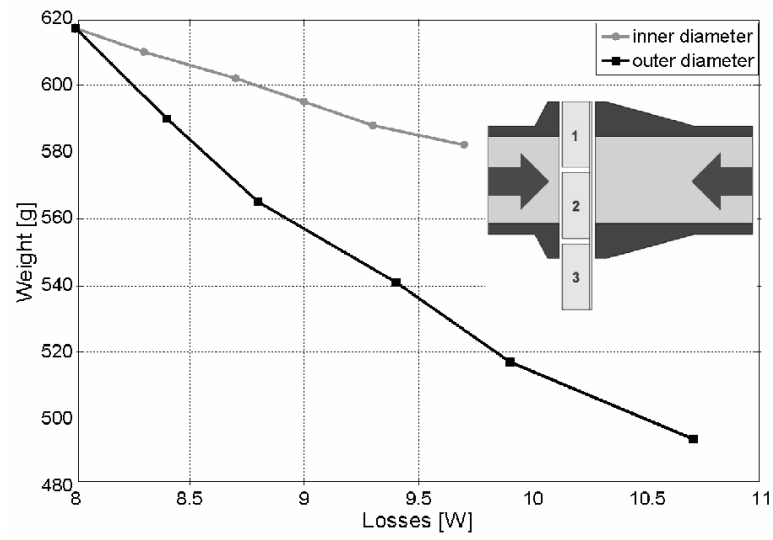

Fig. 4. Variation of inner and outer diameter

\subsection{Parameter variation}

The linear drive is optimised with a parameter variation. In a first step the parameters inner and outer diameter, the coil and therefore the air gap thickness, as well as the inner diameter of the air gap are selected. Then these parameters are varied in fixed steps. By applying the described calculation chain the weight and the average losses for one pumping cycle are calculated for each variant. A comparison of the results yields the coupling sensitivity between weight and losses. In Figure 4 the weight vs. losses diagram for variations in steps of $2 \mathrm{~mm}$ of the inner and outer diameter of the TAH drive is shown. At constant losses, the variation of the outer diameter yields a higher reduction of weight compared to variations of the inner diameter. For this reason, in further optimisation only the outer diameter is varied whereas the inner diameter is fixed to its initial value. In Figure 5, the results of the reduction of the outer diameter for a radial coil thickness of $2 \mathrm{~mm}$ and $3 \mathrm{~mm}$ are illustrated.

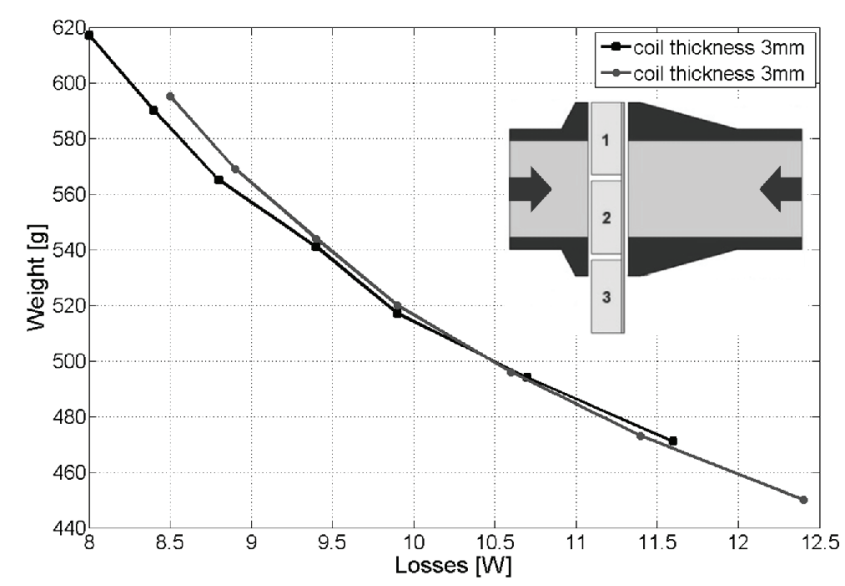

Fig. 5. Variation of the coil thickness

While the average coil radius stays constant, the radial dimensions of the coils are symmetrically reduced. The increased air gap is filled by a radial scaling of the magnets and the pole 
shoes. Thus, a reduction of the drive's weight is achieved, because the material density of the copper wire $\left(8.92 \mathrm{~g} / \mathrm{cm}^{3}\right)$ is higher than for the $\mathrm{NdFeB}$ magnets $\left(7.4 \mathrm{~g} / \mathrm{cm}^{3}\right)$ and for the iron vanadium cobalt alloy $\left(8.12 \mathrm{~g} / \mathrm{cm}^{3}\right)$ used for the pole shoes. Additionally, the axial height of the coils amounts about twice the magnets and pole shoes height, decreasing the weight further. On the other hand, the coil's resistant is increasing due to the reduced cross section of the coils, downgrading the drive's losses. This effect is partially compensated by the reduction of the leakage flux, concentrating the flux density in the air gap more effective. These complex correlations yield the intersection between the two curves in the range of $10.6 \mathrm{~W}$ of losses and $500 \mathrm{~g}$ weight. When decreasing the outer diameter even more, the losses are lower for a coil thickness of $2 \mathrm{~mm}$. Thus, the next steps of optimization are performed with a reduced coil thickness of $2 \mathrm{~mm}$. In Figure 6 the average diameter of the coils is reduced in steps of 2 and $4 \mathrm{~mm}$. Thus, a small increase of the volume of the permanent magnets is performed while simultaneously decreasing the magnetic resistance of the air gap, resulting in an increase of air gap induction. Furthermore, the coil's resistance is reduced, improving the ohmic losses. On the other hand the active wire length $l$ is decreasing, causing an increase of coil current to achieve the required forces. Also in this case, an intersection between the three curves is observed, indicating when it is advisable to change the inner diameter of the air gap.

When comparing the three presented radial optimisation options, the lowest achievable weight is $450 \mathrm{~g}$. In this case the outer diameter is decreased by $12 \mathrm{~mm}$ and the coil thickness amounts to $2 \mathrm{~mm}$. For providing the required forces, the average ohmic losses of the coils amount to $12.2 \mathrm{~W}$.

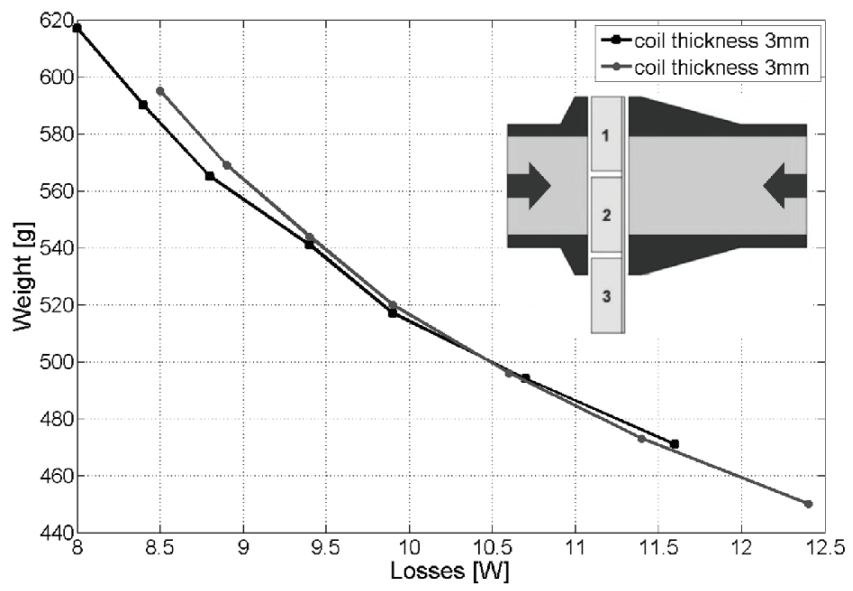

Fig. 6. Variation of the average coil diameter

Table 1. Comparison of prototype and optimiesed geometry

\begin{tabular}{l|c|c|c}
\hline \multicolumn{1}{c|}{ Parameter } & Prototype & Optimised Drive & Variation (\%) \\
\hline Outer diameter & $85 \mathrm{~mm}$ & $73 \mathrm{~mm}$ & $-14.1 \%$ \\
Coil thickness & $3 \mathrm{~mm}$ & $2 \mathrm{~mm}$ & $-33 \%$ \\
Drive weight & $616 \mathrm{~g}$ & $450 \mathrm{~g}$ & $-26.9 \%$ \\
Losses & $8 \mathrm{~W}$ & $12.2 \mathrm{~W}$ & $+52.5 \%$ \\
\hline
\end{tabular}




\section{Results}

Table 1 summarizes the results of the optimisation, which is based on an evaluation of the parameter variation. In order to achieve the required force vs. displacement curve for nominal operation, the average ohmic losses of the actual TAH prototype drive only amount to $8 \mathrm{~W}$ at a weight of $617 \mathrm{~g}$. Nevertheless, previously performed thermal measurements yielded maximum permitted average losses of up to $20 \mathrm{~W}$. Therefore, increasing the losses by $4.2 \mathrm{~W}$ allows to reduce the drive's weight by $167 \mathrm{~g}$. Thus the final weight of the drive achieved by this optimisation is $450 \mathrm{~g}$.

\section{Conclusions}

The requirements and limitations of a totally implantable TAH for the therapy of terminal heart diseases were discussed. An innovative TAH prototype, based on a linear drive, already achieves all requirements except the weight. Exemplaraly presented measurements, performed on the left blood chamber of the TAH in in-vitro tests, yielded the required force vs. displacement characteristic to achieve a sufficient blood perfusion of the human body, i.e. a flow rate of $61 / \mathrm{min}$ at an average pressure of $100 \mathrm{mmHg}$ in the arterial blood circuit. Based on this characteristic a parameter variation was performed to reduce the weight of the drive while limiting the losses as much as possible. In order to reduce the computation time without reducing computation accuracy, a combination of analytical and numerical approaches was chosen. Due to the non linear B-H curve of the soft magnetic pole shoes and the significant magnetic flux leakage FE-simulations are applied to calculate the distribution of the flux density in the linear drive. Then an optimum current supply of the moving coils is determind for providing the required force vs. displacement characteristics by applying the Lorentz force equation. Based on the time dependent current distribution, the average losses of the drive during one beat cycle have been computed analytically. Optimisation results yielded a reduction of the weight from $617 \mathrm{~g}$ of the actual TAH drive to $450 \mathrm{~g}$. Under the worst case assumption, that the weight of the housingand the blood pump stays constant, the resulting weight of the TAH amounts to $972 \mathrm{~g}$, exceeding the maximum tolerable weight of $800 \mathrm{~g}$. Butthe reduced outer diameter of the drive enables a design of the housing with reduced outer dimensions and weight. Although the average losses have been increased from $8 \mathrm{~W}$ to $12.2 \mathrm{~W}$, the loss limit of $20 \mathrm{~W}$ has not been completely exploited. Therefore a further optimisation of the drive geometry, yielding an increase in the losses of the drive, is possible. Due to the dependency of the axial coil height on the axial height of the permanent magnets and pole shoes, variations in axial dimensions are not performed in this paper. For this reason these parameters should be included in the next optimization step.

\section{References}

[1] Abstracts from the $14^{\text {th }}$ Congress of the International Society for Rotary Blood Pumps. Artif. Organs, 30(11): A 27 (2006). 
[2] Lessmann M., Finocchiaro T., Steinseifer U. et al., Concepts and designs of life support systems. IET Science, Measurement \& Technology 2(6): 499-505 (2008).

[3] Lu H., Zhu J., Lin Z., Guo Y., A miniature short stroker linear actuator - design and analysis. IEEE Transactions on Magnetics 44: 497-504 (2008).

[4] Vacuumschmelze, www.vacuumschmelze.de, accessed in April 2010.11.

[5] Institute of Electrical Machines, www.iem.rwth-aachen.de, accessed in May 2010, rWTH Aachen University. 